\title{
Functional variants at the $21 q 22.3$ locus involved in breast cancer progression identified by screening of genome-wide estrogen response elements
}

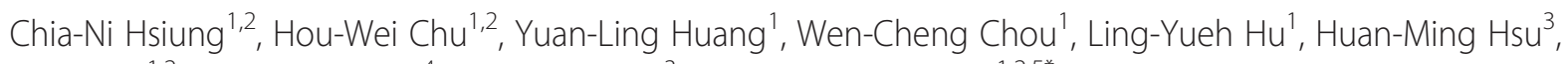
Pei-Ei Wu ${ }^{1,2}$, Ming-Feng Hou ${ }^{4}$, Jyh-Cherng $\mathrm{Yu}^{3}$ and Chen-Yang Shen ${ }^{1,2,5^{*}}$

\begin{abstract}
Introduction: Estrogen forms a complex with the estrogen receptor (ER) that binds to estrogen response elements (EREs) in the regulatory region of estrogen-responsive genes and regulates their transcription. Sequence variants in the regulatory regions have the potential to affect the transcription factor-regulatory sequence interaction, resulting in altered expression of target genes. This study explored the association between single-nucleotide polymorphisms (SNPs) within the ERE-associated sequences and breast cancer progression.
\end{abstract}

Methods: The ERE-associated sequences throughout the whole genome that have been demonstrated to bind ERa in vivo were blasted against online information from SNP data sets and 54 SNPs located adjacent to estrogen-responsive genes were selected for genotyping in two independent cohorts of breast cancer patients: 779 patients in the initial screening stage and another 888 in the validation stage. Deaths due to breast cancer or recurrence of breast cancer were defined as the respective events of interest, and the hazard ratios of individual SNPs were estimated based on the Cox proportional hazards model. Furthermore, functional assays were performed, and information from publicly available genomic data and bioinformatics platforms were used to provide additional evidence for the associations identified in the association analyses.

Results: The SNPs at 21q22.3 ERE were significantly associated with overall survival and disease-free survival of patients. Furthermore, these 21q22.3 SNPs (rs2839494 and rs1078272) could affect the binding of this ERE-associated sequence to ERa or Rad21 (an ERa coactivator), respectively, which resulted in a difference in ERa-activated expression of the reporter gene.

Conclusion: These findings support the idea that functional variants in the ERa-regulating sequence at 21q22.3 are important in determining breast cancer progression.

\section{Introduction}

The roles of estrogen receptor $\alpha(E R \alpha)$ in initiating tumor development in breast cancer, regulating progression and determining therapeutic protocols and efficacy are well documented [1,2]. However, not all patients with the same ER $\alpha$ status manifest the same cancer progression or response to hormone therapy, and individual

\footnotetext{
* Correspondence: bmcys@ibms.sinica.edu.tw

'Institute of Biomedical Sciences, Academia Sinica, Academy Street, Taipei 115, Taiwan

${ }^{2}$ Taiwan Biobank, Academia Sinica, Academy Street, Taipei 115, Taiwan Full list of author information is available at the end of the article
}

variations in breast cancer progression have remained an issue of particular concern. Although ER $\alpha$ can be activated in an estrogen-independent manner, the classical activation mechanism involves the binding of ER $\alpha$ to estrogen and other coactivator proteins to form the estrogenbound ER complex, which functions as a transcriptional regulator $[3,4]$. The DNA-binding domain of ER $\alpha$ binds to estrogen response elements (EREs) in the regulatory region of estrogen-responsive genes, activating or repressing their transcription and consequently mediating physiological or tumorigenic effects. Since sequence variants, 
such as single-nucleotide polymorphisms (SNPs), located in the regulatory regions of genes have the potential to affect protein (transcription factor)-DNA (regulatory region) interactions, resulting in altered expression of target genes $[5,6]$. We previously examined the hypothesis that genetic variations of genome-wide EREs might be associated with breast cancer development, and we identified a significant effect of several ERE-associated SNPs on breast cancer risk [7]. However, because the ERE sites we examined were based on prediction by a computational algorithm and lacked confirmation by results from cell-based assays, it was not possible to know whether such EREs indeed function as predicted in vivo. In the present study, we explored the association between genetic variants within these ERE-associated sequences and breast cancer progression. Importantly, these ERE-associated sequences scattered throughout the whole genome have been shown, by using chromatin immunoprecipitation (ChIP)-based methods, to bind ER $\alpha$ in vivo [8-10]. This is a promising approach for identifying the breast tumorigenic contribution of EREs on a genome-wide scale. Furthermore, we performed functional assays and used information from publicly available genomic data and bioinformatics platforms to provide additional evidence for the association identified in the association analysis. The results obtained by the combined use of these different approaches in this multistage study support the idea that functional variants in the ER $\alpha$-regulating sequence at 21q22.3 are important in determining breast cancer progression.

\section{Methods}

\section{Study participants}

Two independent cohorts of patients with incident primary breast cancer, 779 of whom were in the initial screening stage and another 888 in the validation stage (Figure 1A), were included in the present study. All of the patients were part of our ongoing cooperative study aimed at understanding the causes and progression of breast cancer in Taiwan. Their characteristics have been described in detail elsewhere [7,11-13]. Two independent groups of women without a history of cancer were recruited. One of these groups comprised 870 women from the same source as the patients in the initial screening phase. That group was used as the control group to explore the association between SNPs and breast cancer development. The other group comprised 903 women chosen from the National Biobank of Taiwan [14]; we used their data to provide background information about haplotype block and linkage disequilibrium (LD) between SNPs in our population.

This study was approved by the ethics committee of the institutional review board of the Academia Sinica, Taiwan, and informed consent was obtained from all study participants before the collection of data by personal interview.

\section{Single-nucleotide polymorphisms and genotyping}

Genome-wide EREs were detected by ChIP using anti$E R \alpha$ antibodies in different ER $\alpha$-positive breast cancer cells [8-10]. These ERE sites were blasted against the SNP database, resulting in the identification of the EREassociated SNPs. Multiple steps were used to select the SNPs for genotyping; these steps are described in the Results section. In the initial screening stage, SNPs were genotyped in all samples tested using Sequenom iPLEX technology (Sequenom, Hamburg, Germany). Duplicate positive and negative controls were included on all plates, with genotypes autocalled by using specialized software (MassARRAY Typer version 3.4; Sequenom) and subsequently confirmed by visual assessment of the data. All assays were performed by individuals blinded to the case versus control status of the samples. As a quality control, we repeated the genotyping on $10 \%$ of the samples, and all genotype scoring was performed and checked separately by one reviewer who was unaware of the case versus control status. The concordance rate for replicate samples was $100 \%$. In the validation stage, the genotyping data at specific SNPs for 888 patients with incident breast cancer were used. These SNPs showed strong LD with those significant SNPs identified in the initial screening stage. These 888 patients had been included in the international Collaborative Oncological Gene-environment Study (COGS), in which genotyping was performed using a customized Illumina Infinium BeadChip array (Illumina, San Diego, CA, USA) [15,16]. Using the National Biobank of Taiwan, a total of 642,832 SNPs from 903 women were genotyped, and the details of the SNPs, how they were selected and the genotyping results are publicly available on the Taiwan Biobank website [14].

\section{Statistical analysis}

To identify putative high-risk genotypes of ERE-associated SNPs for breast cancer incidence in the initial screening stage, we followed our previously established sequential steps $[7,11-13]$ to analyze the data. Hardy-Weinberg equilibrium was tested to ensure that the chance of genotyping error was small. Logistic regression was used to estimate the odds ratio of breast cancer associated with harboring an individual genotype. Data on ER $\alpha$ status (positive or negative), histologic grade (well-differentiated to poorly differentiated) and clinical stage (I to IV) were collected from hospital medical records. We were thereby able to determine whether an ERE-associated SNP influenced prognosis. To this end, we calculated the overall survival (OS) rate and the breast cancer-specific survival rate (that is, disease-free survival (DFS)) using the Kaplan-Meier method and the two-tailed logrank test, with death due to 


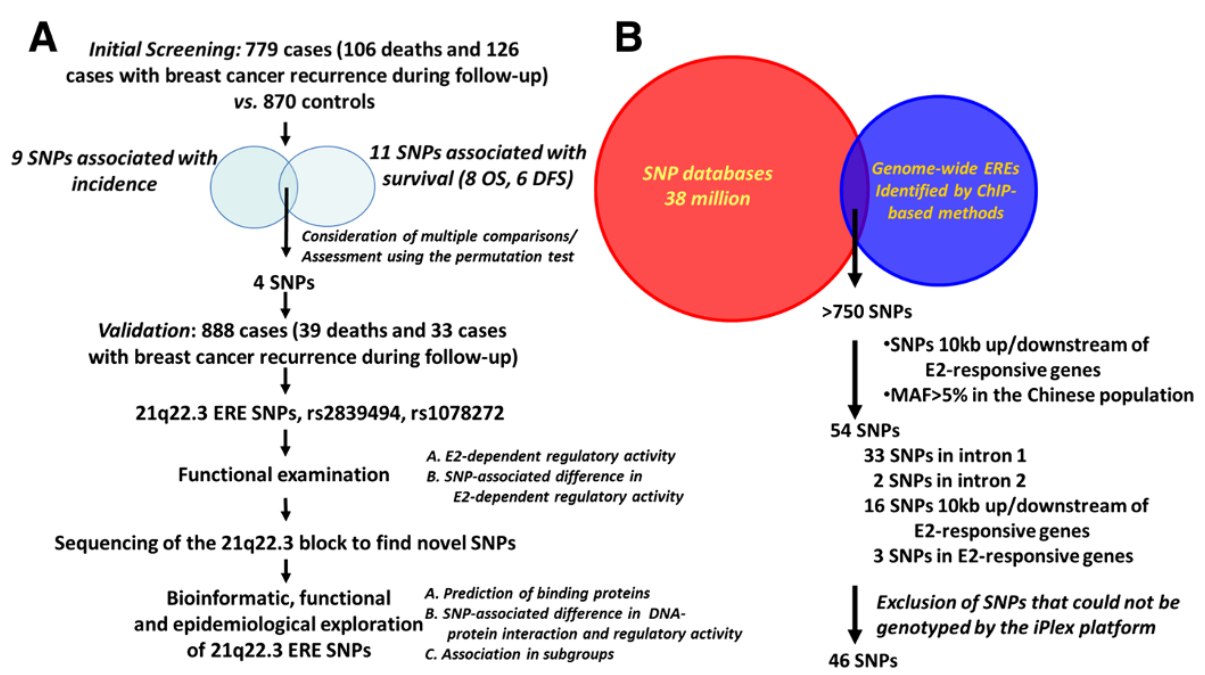

Figure 1 Identification and functional examination of genome-wide estrogen response element-associated single-nucleotide polymorphisms associated with breast cancer progression. (A) Flow diagram showing the sequential steps in the present study. (B) Selection of single-nucleotide polymorphisms (SNPS) to be genotyped. SNPs located within regions that bind estrogen receptor a (ERa) in vivo were selected, and then they were examined for whether they were located within the regions $10 \mathrm{~kb} 3^{\prime}$ or $10 \mathrm{~kb} 5^{\prime}$ of known estrogen (estradiol (E2))-responsive genes. As a result, after excluding those that could not be genotyped by using the iPLEX platform, 46 estrogen response element (ERE)-associated SNPs were genotyped. ChIP, Chromatin immunoprecipitation; DFS, Disease-free survival; OS, Overall survival.

breast cancer or recurrence of breast cancer defined as the respective event of interest. In addition, the hazard ratios (HRs) of individual SNPs associated with OS or DFS were estimated based on the Cox proportional hazards model, considering the effect of the patients' age, ER status and cancer stage. All statistical analyses were performed using SAS version 9.1 software (SAS Institute, Cary, NC, USA), and all tests were based on a two-sided probability. To address the issue of falsepositivity due to multiple tests, a permutation test [17] was performed when needed.

LD plots of the D' values for SNPs within the same haplotype block were produced using the Haploview program [18]. Haplotype estimation was performed on individuals for whom complete genotype data were available across all polymorphic sites, and the highest probability haplotypes, estimated using the expectation maximization algorithm of SAS Genetics 9.1 (SAS Institute), were assigned to each study participant [19].

\section{Sequencing}

Sanger sequencing was performed to detect singlenucleotide variants within specific genomic regions.

\section{Cell culture}

The human breast cancer cell line MCF-7 was purchased from the Taiwan Bioresource Collection and Research Center. The cells were cultured in Dulbecco's modified Eagle's medium (Sigma-Aldrich, St Louis, MO, USA), supplemented with $10 \%$ fetal bovine serum (Gibco, Grand Island, NY, USA).

\section{Construct generation, transfection and site-directed mutagenesis}

The ER $\alpha$-binding regions containing specific ERE-associated SNPs were amplified by PCR from human genomic DNA and inserted into the promoter region of the pGL3-Basic vector (Promega, Madison, WI, USA) at the NheI-XhoI restriction site. ER $\alpha$ - and Rad21-expressing constructs were amplified from cDNA. The former were cloned into the EcoRI-XhoI sites, and the latter into the NotI-XhoI sites, of the mammalian expression vector pcDNA3-Flag. For site-directed mutagenesis, specific point mutations (that is, variant alleles) were generated using QuikChange Site-Directed Mutagenesis kits (Stratagene/ Agilent Technologies, Santa Clara, CA, USA) according to the manufacturer's instructions. Transfection of plasmids was performed using Lipofectamine 2000 reagent (Invitrogen, Carlsbad, CA, USA) according to the manufacturer's instructions.

\section{Luciferase reporter assay}

The luciferase constructs and pRL-TK, which encodes Renilla luciferase, were cotransfected into $5 \times 10^{4} \mathrm{MCF}-7$ cells in 24-well plates. After 48 hours, the cells were lysed in a single freeze-thaw cycle in passive lysis buffer. The lysate was then centrifuged at $12,000 \mathrm{~g}$ for $10 \mathrm{mi}$ nutes at $4^{\circ} \mathrm{C}$, and the luciferase activities in the supernatant were measured using Dual-Luciferase Reporter Assay System kits (Promega). The relative activity of luciferase was determined using the Renilla luciferase signal as the reference. 


\section{Chromatin immunoprecipitation}

ChIP was performed based on our previous protocol [20] using EZ-Magna ChIP G kits (EMD Millipore, Billerica, MA, USA) following the manufacturer's instructions and using anti-Flag monoclonal antibody (F3165; Sigma-Aldrich) for the precipitation stage. The immunoprecipitate was eluted with $50 \mu \mathrm{l}$ of the supplied Tris-ethylenediaminetetraacetic acid buffer, and $2 \mu \mathrm{l}$ of DNA were used in quantitative PCR (qPCR). The primer pairs used for the ChIP PCR were 5 '-CCGGCCATCTCTC ACTATGAA-3' and 5'-CCTTCCCGCCAGGGTAAATA C-3' for TFF1 and 5'-CTTGAGGTGCTTCGAGACAGT G-3' and 5'-CACCTGCTTCAAAGTGAGTGAG-3' for 21q22.3.

\section{Results}

\section{Characteristics of the patient cohorts}

The risk profile of breast cancer in our study participants was similar to that found in our previous studies [11-13] and in other breast cancer studies [21]. Development of breast cancer was found to be highly associated with reproductive risk factors, including early menarche (adjusted odds ratio (aOR), 1.33; 95\% confidence interval (CI), 1.09 to 1.64 ), nulliparity (aOR, $1.37 ; 95 \% \mathrm{CI}, 1.03$ to 1.85), low number of full-term pregnancies (less than two) (aOR, 1.12; 95\% CI, 0.89 to 1.40 ) and no history of breastfeeding (aOR, 1.49; 95\% CI, 1.21 to 1.83). Importantly, these significant associations between reproductive risk factors and breast cancer reveal the importance of the estrogen-related etiology of breast cancer in our participants, providing us with the opportunity to examine the contribution of EREs during breast tumorigenesis. Because the present study was focused on SNPs in EREassociated sequences involved in determining breast cancer progression, we also examined factors in the clinical profile that correlated with OS and DFS. As expected, patient age at tumor onset, ER status and tumor stage were three major determinants in our cohort and were included in all of our analyses so that we could examine the effect of SNPs on breast cancer progression.

\section{Selection of estrogen response element-associated single-nucleotide polymorphisms for genotyping}

Genome-wide ER $\alpha$-binding sites have previously been detected using ChIP-based methods [8-10], and more than 1,500 binding regions have been identified. After blasting these regions against online information available from SNP data sets (UCSC Genome Browser, National Center for Biotechnology Information and HapMap databases), including more than 38 million SNPs throughout the whole genome, more than 750 SNPs were identified in ER $\alpha$-associated sequences (Figure 1B). We next examined whether these SNPs were located adjacent to estrogenresponsive genes, as genetic variants near genes may affect the interactions of transcription factors with the promoter/enhancer/regulatory regions, resulting in altered mRNA expression. To do so, we used the bioinformatics tool GenePipe [22] to screen for SNPs located within the regions $10 \mathrm{~kb} \mathrm{3'}$ or $10 \mathrm{~kb} \mathrm{5'}$ of estrogen-responsive genes, identified by showing a significant change in expression when the ER $\alpha$-positive breast cancer cells were treated with estrogen [8-10]. Taking statistical power considerations into account, we included only SNPs with a minor allele frequency greater than $5 \%$ in the Chinese population. As a result, 54 SNPs were identified, and, after excluding 8 that could not be genotyped in the iPLEX platform, a total of 46 SNPs were genotyped in all patients and controls in the initial screening stage (Figure 1A).

Identification of single-nucleotide polymorphisms associated with breast cancer development and progression

We next sought to determine the breast tumorigenic contribution of ERE-associated SNPs. In the initial screening stage, we examined whether the genotypic distribution of these 46 SNPs differed between cases and controls and between cases with different progression outcomes (that is, OS versus DFS) (Figure 1A). The frequencies of all SNPs in the controls agreed with those expected on the basis of the Hardy-Weinberg equilibrium, suggesting that genotyping errors were relatively unlikely. The results for the genotypic analysis showed that nine SNPs were associated with breast cancer incidence (Figure $2 \mathrm{~A}$ and 2B, left panel) and that eleven SNPs were associated with survival (DFS or OS) (Figure $2 \mathrm{~A}$ and $2 \mathrm{~B}$, center and right panels). Women carrying the homozygous variant genotype had a significantly increased aOR or HR $(P<0.05)$ compared to women carrying the homozygous and heterozygous wildtype genotypes. The possibility of false-positives due to multiple testing is relatively unlikely, because the results of the permutation test [17], based on 10,000 random permutations, showed that these associations were significant (data not shown). Particular attention was focused on four SNPs, each of which was associated with both breast cancer incidence and progression (Figures 1A, 2A and 2B). Though the genetic variants associated with cancer progression are not necessarily those associated with cancer incidence, variants that play a dual role in different stages during tumorigenesis are certainly of more tumorigenic importance. Of these four SNPs, two (rs2839494 and rs1078272) are located in the same LD block at 21q22.3 and the other two at 5p12 and 20q13.2 (Figure 2A).

\section{Validation of 21 q22.3 single-nucleotide polymorphisms for breast cancer progression}

To confirm the significant associations between specific ERE-associated SNPs and breast cancer progression detected in the initial screening, we studied an independent cohort of 888 breast cancer patients (Figure 1A). 


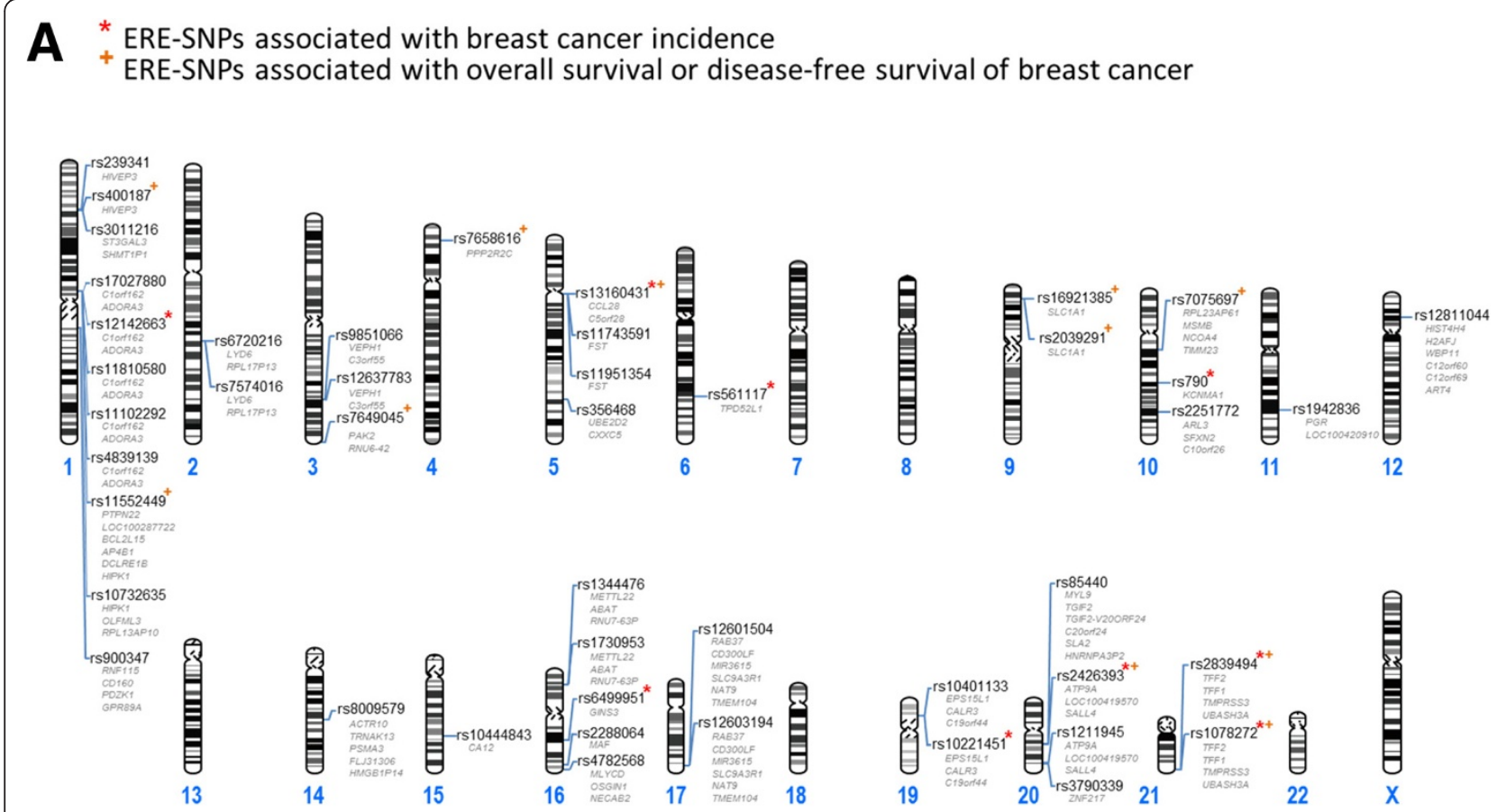

B

\begin{tabular}{l|l|l} 
SNP & aOR $(95 \% \mathrm{Cl})$ \\
\hline$r s 13160431$ & \\
$r s 561117$ & \\
$r s 1078272$ & \\
$r s 12142663$ & \\
$r s 2839494$ & \\
$r s 2426393$ & \\
$r s 6499951$ & \\
$r s 790$ &
\end{tabular}
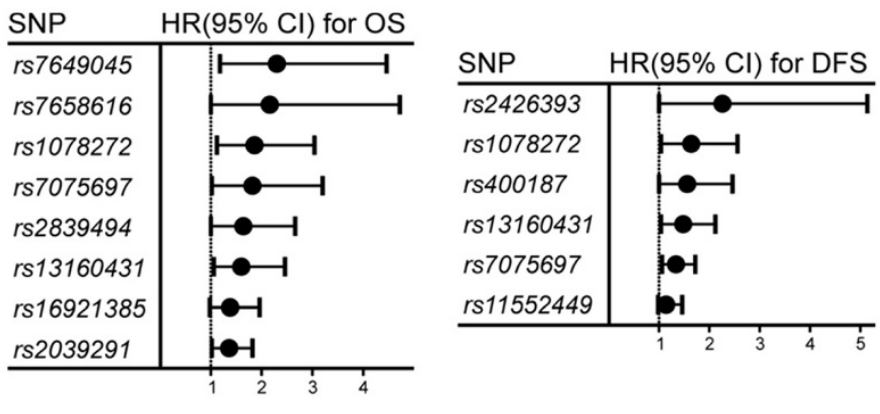

Figure 2 Estrogen response element-associated single-nucleotide polymorphisms related to breast cancer incidence and progression. Genomic sites (A) and adjusted odds ratios (aORs), hazard ratios (HRs) and corresponding 95\% confidence intervals (Cls) (B) of the estrogen response element (ERE)-associated single-nucleotide polymorphisms (SNPs) found to be significantly associated with breast cancer incidence (left panel), overall survival (OS; center panel) or disease-free survival (DFS; right panel). Known genes adjacent to these SNPs are shown in gray in (A). aORs were estimated by logistic regression analyses, and the HRs were estimated by applying the Cox proportional hazards model. In total, 779 patients (106 deaths and 126 cases with breast cancer recurrence during follow-up) and 870 controls were included in these analyses.

The genotyping data for these patients were recently reported in the COGS genome-wide association study (GWAS) $[15,16]$. We first validated that the SNPs at $5 \mathrm{p} 12$, 20q13.2 and 21q22.3, genotyped in our COGS patients, were in very strong LD (all LD coefficients between SNPs $>0.95$ in our population) with the four significant SNPs identified in our initial screening, not only in Han Chinese using HapMap data but also in Taiwanese women using Taiwan Biobank data [14]. These SNPs were then tested for their associations with cancer progression. The polymorphic status of 21q22.3, now reflected by rs2251362 (Figure 3A), remained significantly associated with OS and DFS in the validation stage (Figure 4A). More importantly, to examine whether an ER $\alpha$-associated mechanism could explain this association, we stratified our patients based on the ER status of their tumors and found that the significant association between rs2251362 and cancer progression was present only in the ER $\alpha$-positive patients (Figure 4A). These results prompted us to retrospectively check the interaction between 21q22.3 SNPs and the ER in the patients included in the initial screening. Consistent with the finding above, the association between breast cancer progression (indicated by OS) and ERE-associated SNP at 21q22.3 (that is, rs1078272) was more significant in patients harboring specific genotypes of a SNP (rs985694) of ESR1, 


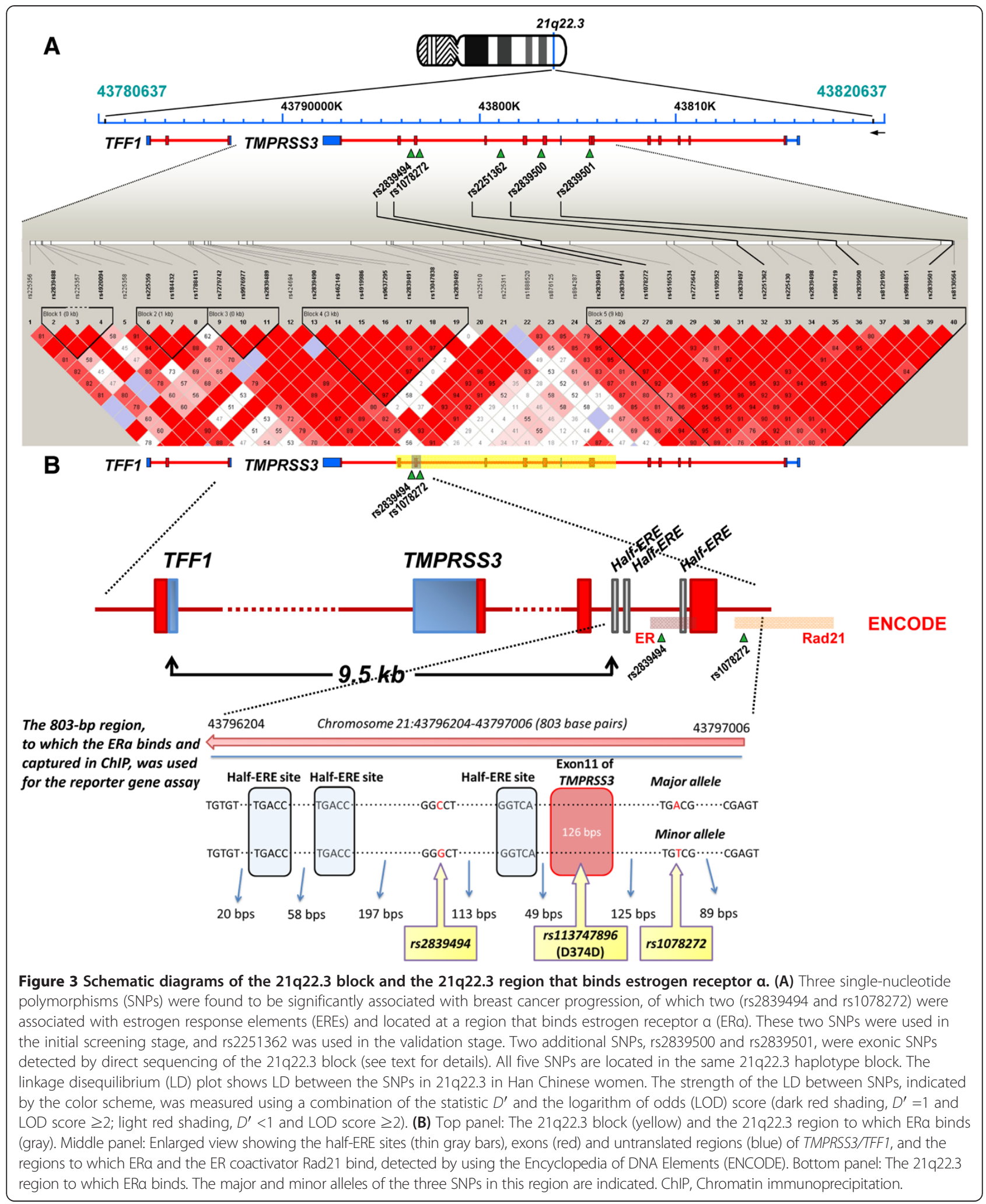

the gene encoding ER $\alpha$ (Figure 4B). We also performed haplotype analysis in which a polymorphism of 21q22.3 was defined more precisely by the two ERE-associated
SNPs rs2839494 and rs1078272. As shown in Figure 4C, women carrying the haplotype pair of the variant allele of both rs2839494 and rs1078272 (Vt-Vt/Vt-Vt) were 

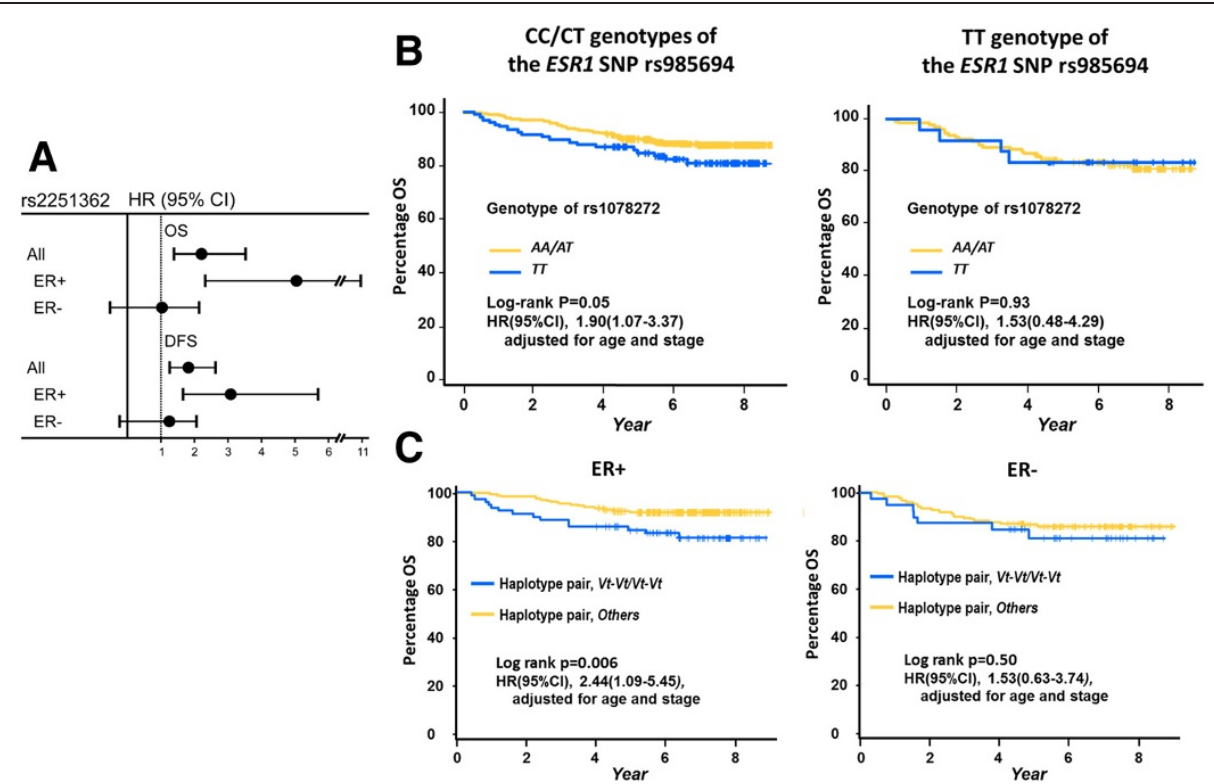

Figure 4 Associations between single-nucleotide polymorphisms in 21q22.3 in tumors displaying different estrogen receptor status and breast cancer progression. (A) The 21q22.3 single-nucleotide polymorphism (SNP) rs2251362 is significantly associated with both overall survival (OS) and disease-free survival (DFS) in all patients, especially in estrogen receptor-positive (ER+) patients, as detected in the validation stage. Hazard ratios (HRs) and 95\% confidence intervals ( $95 \% \mathrm{Cls}$ ) were estimated based on the Cox proportional hazards model, considering the effects of the patients' age, ER status and cancer stage. A total of 888 breast cancer patients (39 deaths and 33 patients with breast cancer recurrence during follow-up) were included. (B) The association between genetic polymorphism of the estrogen response element (ERE)-associated SNP in 21q22.3 (defined by rs1078272) and OS was significantly modified by the genotype of the SNP (that is, rs985694) of ESR1, the gene encoding the ER. (C) Association between genetic polymorphism of 21q22.3 detected by the haplotype pairs containing the two ERE-associated SNPs (rs2839494 and rs 1078272) and OS of breast cancer patients with ER + tumors (left panel) or ER - tumors (right panel). The 779 patients (106 deaths and 126 patients with breast cancer recurrence during follow-up) in the initial screening were included in the analyses depicted in (B) and (C). HRs and 95\% Cls were estimated based on the Cox proportional hazards model, considering the effects of the patients' age and cancer stage.

found to manifest a significantly worse survival than those carrying other haplotype pairs, particularly in the ER-positive patients.

\section{Functional examination of the two estrogen response element-associated single-nucleotide polymorphisms at $21 \mathrm{q} 22.3$}

On the basis of the above-described results, functional studies were performed to examine the effect of these two ERE-associated SNPs at 21q22.3 (Figure 1A). As shown in Figure 3B, rs2839494 and rs1078272 are located in an ER $\alpha$-binding segment spanning $803 \mathrm{bp}$, containing three half-ERE sequences and covering exon 10 of TMPRSS3. In addition, at a region $9.5 \mathrm{~kb} \mathrm{5} 5^{\prime}$ of this segment, there is a gene, TFF1, which has been shown to be a suppressor of breast cancer in a mouse model [23]. We first demonstrated estradiol (E2)-dependent regulatory activity of this 803-bp segment by an E2 dose-dependent increase in reporter gene activity when the segment was linked to the luciferase reporter gene, the construct transfected into ERpositive MCF-7 cells and the transfected cells incubated with increasing levels of E2 (Figure 5A). To examine the effect of the variant alleles on transcription and to differentiate the effects of rs2839494 and rs1078272, we generated one variant allele of each SNP by mutating the wild type and tested their individual effects in regulating reporter gene activity. Each of the variant alleles led to significantly decreased luciferase activity (Figure 5B). However, interestingly, the two variant alleles resulted in different phenotypes with decreased ER $\alpha$-regulated activity. The variant allele of rs2839494 completely abolished the response to E2, and the variant allele of rs1078272 decreased the response but maintained the dose-response relationship between E2 and reporter gene activity. This marked difference suggested different inhibition mechanisms (Figure 5C), which were examined in our following experiments.

\section{Direct sequencing to search for additional single-nucleotide polymorphisms within 21q22.3}

To gain support for a causal role of these two EREassociated SNPs (rs2839494 and rs1078272) in 21q22.3, we attempted to clarify alternative possibilities, including that (1) rs2839494 and rs1078272 might be in LD with an exon change in TMPRSS3 that affects protein function, (2) other changes in this $21 \mathrm{q} 22.3$ block might be within regulatory sequences and affect the level of expression through transcriptional regulation and (3) alterations in other adjacent genes might increase susceptibility to breast cancer 


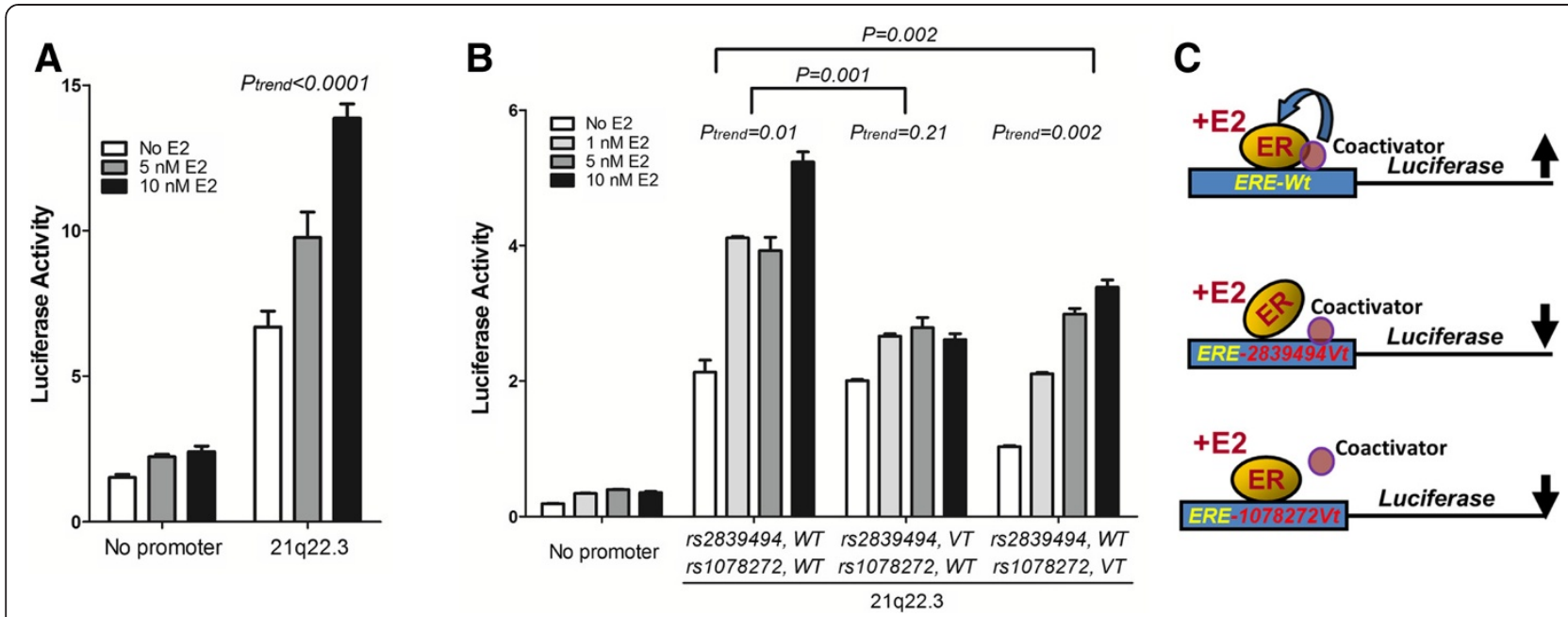

Figure 5 Functional examination of the two estrogen response element-associated single-nucleotide polymorphisms rs2839494 and rs1078272 in 21q22.3. (A) Activity in MCF-7 cells of the luciferase reporter gene, either alone (denoted as "No promoter") or linked to the 21 q22.3 region (that is, the segment shown in Figure 3B, bottom panel) to which estrogen receptor a (ERa) binds, and which contains the two estrogen response element (ERE)-associated single-nucleotide polymorphisms (SNPs) (denoted as "21q22.3"), in the presence or absence of estradiol (E2). (B) Decreased activity of the luciferase reporter gene in MCF-7 cells when linked to the 21 q22.3 region harboring the variant allele (VT) of rs2839494 or rs1078272 compared to that containing both wild-type alleles (WT). The results shown in (A) and (B) are means \pm standard deviations $(n=3)$. The $P$-values of differences between groups and the $P$-values for trends within groups were estimated by regression analysis. (C) Hypothetical model explaining the similar, but not identical, effects of the two ERE-associated SNPs at 21q22.3, showing that the variant allele of rs 1078272 might block the binding of a coactivator to the 21q22.3 sequence (bottom panel), whereas the variant allele of rs 2839494 might directly affect the binding of ERa (center panel), both decreasing reporter gene activity compared to the wild-type 21q22.3 sequence (top panel).

progression. Because TMPRSS3 is the only gene in this $21 \mathrm{q} 22.3$ block (Figure 3A and 3B, top panel), the third possibility is unlikely. To examine the first possibility, using blood specimens from 100 healthy individuals, we performed exonic sequencing of all TMPRSS3 exons within this block and identified three SNPs: rs2839500, rs2389501 and rs113747896 (Figure 3A and 3B, bottom panel; and Table 1). None of these SNPs are novel; the first two have been shown to have no pathogenic effect $[24,25]$, and the third, located within exon 10 of TMPRSS3, results in no amino acid change (D374D) (Figure 3B and Table 1). To examine the second possibility listed above, using blood specimens from 58 healthy individuals, we performed direct sequencing to identify any variants within this 21q22.3 block containing 9,940 bp (Table 1). We found that some of the identified SNPs were located at transcription factor binding sites, so we cannot totally exclude the possibility that these variants affect expression of E2/ER $\alpha$-regulating genes. However, it is notable that the 803-bp ERE-associated sequence was the only segment within the 21q22.3 block that could bind ER $\alpha$ (Table 1). As a result, rs2839494 and rs1078272 appear to be candidate causal variants.

\section{Bioinformatics and functional evidence that the} Rad21-coactivated E2-induced increase in promoter activity is affected by ERE-associated SNPs in 21q22.3 It is notable that Encyclopedia of DNA Elements (ENCODE) data [26] show that rs2839494 and rs1078272 lie, respectively, within different binding regions of ER $\alpha$ and $\operatorname{Rad} 21$, a coactivator of ER $\alpha$ [27]. Given the difference in phenotype of the effect on E2-dependent regulatory activity between rs2839494 and rs1078272 (shown in Figure 5B), we speculated that these two SNPs might

Table 1 Direct sequencing of the 21 q22.3 block to identify single-nucleotide variants ${ }^{a}$

\begin{tabular}{|c|c|c|c|c|c|c|c|c|}
\hline & Base pairs & Half-ERE sites & $\operatorname{SNPs}^{\mathbf{b}}(n)$ & $\begin{array}{l}\text { Novel } \\
\text { SNPs }^{\text {b }}(n)\end{array}$ & $\begin{array}{l}\text { Exonic } \\
\operatorname{SNPs}^{c}(n)\end{array}$ & $\begin{array}{l}\text { Novel exonic } \\
\operatorname{SNPs}^{c}(n)\end{array}$ & $\begin{array}{l}\text { SNPs in the TF } \\
\text { binding site }^{d}(n)\end{array}$ & $\begin{array}{l}\text { Binding to ERa } \\
\text { detected by ChIP }\end{array}$ \\
\hline 21q22.3 block & 9,940 & 17 & 37 & 6 & 3 & 0 & 10 & Only the one below \\
\hline $\begin{array}{l}\text { ERa-binding sequence } \\
\text { within } 21 \text { q22.3 }\end{array}$ & 803 & 3 & 3 & 0 & 1 & 0 & 2 & Yes \\
\hline
\end{tabular}

${ }^{a}$ ChIP, Chromatin immunoprecipitation; ERa, Estrogen receptor a; ERE, Estrogen response element; SNP, Single-nucleotide polymorphism; TF, Transcription factor; $\mathrm{N}$, the number of nucleotide. ${ }^{\mathrm{b}}$ Based on HapMap CHB (Han Chinese in Beijing, China), dbSNP137 and direct sequencing of blood specimens from 58 individuals. The novel SNPs were identified by the sequencing of 58 individuals. 'Based on HapMap CHB, dbSNP137 and exonic sequencing of blood specimens from 100 individuals, no exonic SNP was identified. ${ }^{\mathrm{d}}$ Based on Encyclopedia of DNA Elements (ENCODE). The eight SNPs within the TF binding sites but outside the ERa-binding sequence showed no evidence of ERa binding. 
be involved in the protein-DNA interaction (that is, interaction of transcription factors with promoter/enhancer/ regulatory regions) by different mechanisms (Figure 5C).

On the basis of the ENCODE data, rs1078272 is located within the segment bound by $\operatorname{Rad} 21$, and the variant allele might affect the binding of Rad21 to the ERE-associated sequence at $21 \mathrm{q} 22.3$. This suggestion is supported by the finding that, in ChIP assays, the variant allele of rs1078272 led to decreased binding of the ERE-associated sequence to Rad21. An example is shown in Figure 6A, and the pooled results are shown in Figure 6B. Consistent with this hypothesis, as shown in Figure $6 \mathrm{C}$, the dose-dependent increase in relative activity in the luciferase reporter assay caused by Rad21 (left panel) was abolished if this EREassociated sequence contained the variant allele of rs1078272 (right panel).
It is possible that rs2839494 affected the binding of this sequence to ER $\alpha$. This was confirmed by our findings that, in ChIP assays, in addition to binding to Rad21, this ERE-associated sequence at 21q22.3 could bind to ER $\alpha$ (Figure 6D) and that the variant allele of rs2839494 inhibited the binding of this sequence to ER $\alpha$ (Figure 6E). Furthermore, the increase in Rad21 coactivation of the ER-regulated activity caused by E2 was reduced when the wild-type allele of rs2839494 was replaced by the mutant allele (Figure 6F).

These findings prompted us to assess whether Rad21 plays a role in breast cancer progression, particularly via the mechanism proposed above. Interestingly, the SNP rs959692 tagging the Rad21-containing region was not itself associated with OS and DFS, but it did significantly modify the association between SNPs in 21q22.3 and

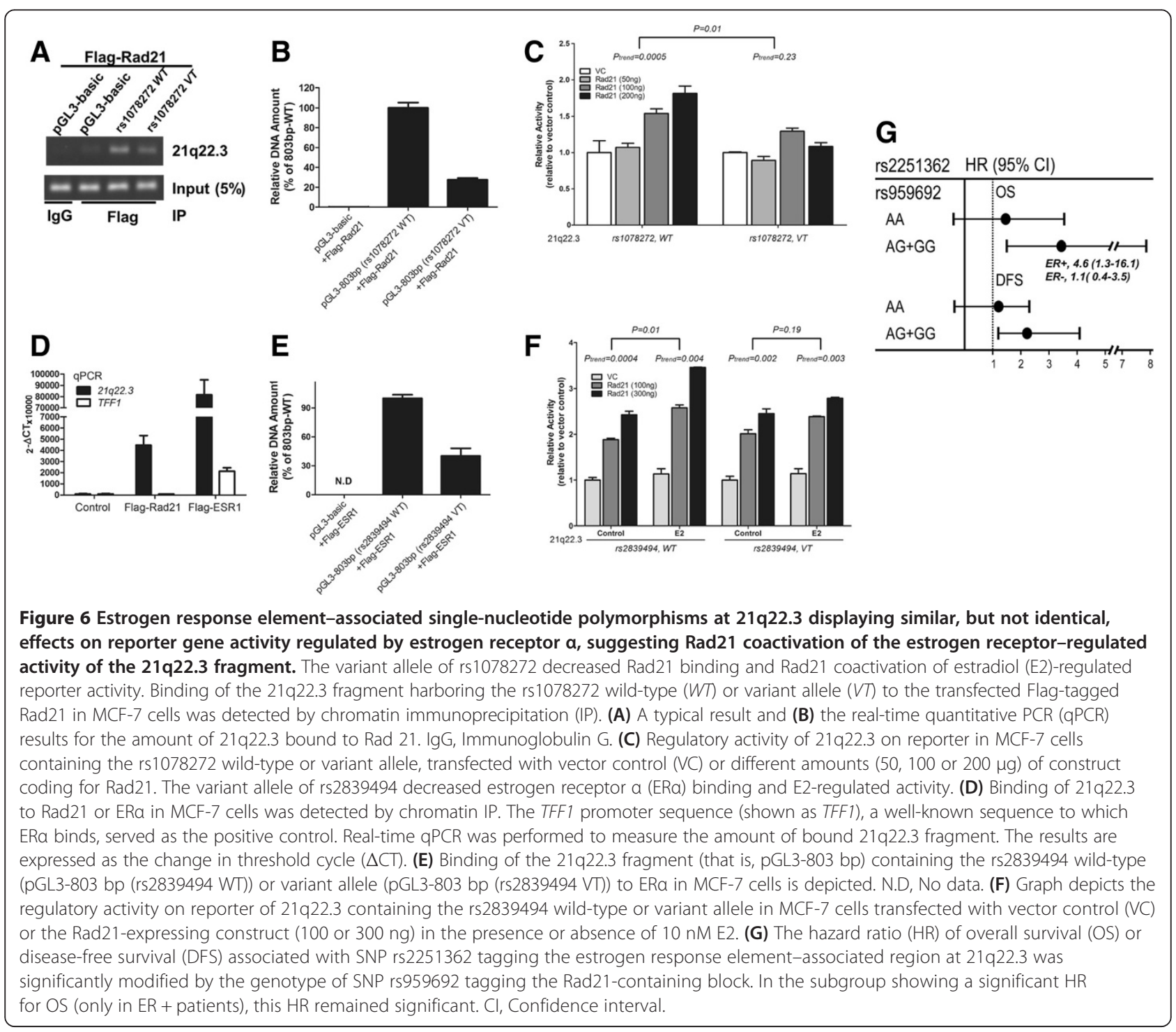


breast cancer progression (Figure 6G). It is notable that, after further subgrouping the patients who showed a significant $\mathrm{HR}$ for OS, this HR remained significant in $E R+$ patients, but not in ER - patients (see ER + and ER - in Figure 6G), a finding in accordance with the results of the cell line-based experiments.

\section{Discussion}

In the present study, we employed two important approaches that make it unique from others. First, in contrast to most genetic and molecular epidemiological studies of breast cancer focused on cancer incidence, we addressed the importance of genetic polymorphisms in determining breast cancer progression. This issue is certainly of more translational relevance, and may add significant prognostic value to the currently used indicators for outcome prediction in breast cancer progression. Second, we employed a novel methodological approach. The move from candidate gene association studies to GWAS has made it possible to explore the etiological contribution of genetic variants throughout the whole genome without relying on an a priori hypothesis. As a result, many novel loci have been identified; the exploration of the genes within these loci should provide information about which genes and biologic pathways are associated with complex diseases. With only a very few notable exceptions, however, the number of detected causal variants directly responsible for individual GWAS associations remains small [28]. Most loci identified by GWAS require further fine-mapping, which usually takes a long time and a tremendous effort. In the present study, to provide a partial solution, we used a hybrid method consisting of candidate gene and genome-wide approaches. The well-defined roles of the ER during breast tumorigenesis make it mechanistically reasonable to assume that polymorphic genetic variants of EREs, central nodes in the ER pathway, might underlie the variations seen between patients in their susceptibility to breast cancer progression. This candidate mechanism lends critical support to the biological plausibility and tumorigenic relevance of our findings. In addition, our genotyping of SNPs on the basis of genome-wide detection of ERE-associated sequences [8-10] provided us a unique opportunity to examine real ER $\alpha$-binding sites comprehensively. The successful identification of two ERE-associated SNPs at 21q22.3 using these combined methods suggests that this is a promising approach which will benefit from the increase in publicly available genomic and epigenomic data and bioinformatics platforms and thus will become more feasible. For example, the ENCODE project [26], in which regions of transcription, transcription factor binding, chromatin structure and histone modification were systematically mapped on a genomewide scale, has generated valuable information that can be combined with SNP database data to address genetic susceptibility to cancer development and progression.

Our reporter gene assay and ChIP results show that the ERE-associated sequence at $21 \mathrm{q} 22.3$ has regulatory activity and that rs2839494 and rs1078272 in this region are able to affect the binding of this sequence to ER $\alpha$ and $\operatorname{Rad} 21$, respectively, resulting in a difference in ER $\alpha$ activated expression of the reporter gene and suggesting that $\operatorname{Rad} 21$ promotes ER $\alpha$-regulated transcription. At the functional level, this is biologically plausible and consistent with the finding that cohesin, a multisubunit protein complex containing Rad21 that is required for activation of transcription of $M y c$ by E2, binds to ER $\alpha$, upon which the complex binds to an ERE 70-kb upstream of Myc [27]. Even though at the molecular level, alternative models remain possible, one of which is that the binding of $\operatorname{Rad} 21$ to rs1078272 is through ER $\alpha$ already bound to this SNP or to the 21q22.3 ERE-associated sequence. However, the finding reported in ENCODE (shown in Figure 3) clearly demonstrates that ER $\alpha$ and $\operatorname{Rad} 21$ can only bind to specific and different segments within this 21q22.3 sequence and that ER $\alpha$-binding segment does not cover rs1078272. Furthermore, we conducted an experiment to show that the effect contributed by the interaction between $\operatorname{Rad} 21$ and the rs1078272-containing sequence to promote expression does not occur indirectly via ER $\alpha$. In this experiment, we used a shorter, 414-bp, rs1078272-containing sequence (that is, the 3' part of the original 803-bp sequence without containing any half-ERE site), and we observed the same result as that shown in Figure $6 \mathrm{C}$, suggesting that the functional interaction between $\operatorname{rs} 1078272$ and $\operatorname{Rad} 21$ is independent of ER $\alpha$. To confirm this hypothesis in further studies, researchers can explore the structure of ER $\alpha$ and $\operatorname{Rad} 21$ bound to DNA and examine whether these two SNPs are located at the protein-DNA interfaces, affecting protein-DNA interaction. Other mechanisms, such as higher-order chromatin-protein interactions, cannot be ruled out.

A more intriguing question is which genes are regulated by the Rad21-promoted, ER $\alpha$-activated mechanism suggested by our findings. We comprehensively checked the region $1,000 \mathrm{~kb} \mathrm{5}$ to $1,000 \mathrm{~kb} \mathrm{3'}$ of rs2839494. The results show that, of the 32 genes in this region (Additional file 1: Figure S1A), 14 have been reported to be involved in various tumorigenic mechanisms (Additional file 1: Figure S1B). Next, on the basis of published information (see, for example $[9,29,30])$, public data sets and the RT-qPCR results in the present study (Additional file 1: Figure S1C), we examined (1) whether expression of these genes has been detected in breast tumor or breast cancer cell lines, (2) whether the expression of these genes is putatively E2dependent and (3) whether the proteins encoded by these genes have a function that is involved in tumor metastasis (Additional file 1: Figure S1B). On the basis of these 
criteria, among E2/ER-responsive genes, TFF2, TFF3 and TMPRSS3 are less likely to be targets of the 21q22.3 EREassociated sequence, because the functions of the proteins encoded by these three genes, as well as clinical observations, suggest that these proteins play a role as metastasis promoters [31,32]. This finding is contrary to what we observed in our patients - that the wild-type alleles of the 21q22.3 SNPs caused increased expression of a reporter gene and were associated with better cancer progression. Next, although it was found to promote migration and invasion in some cell line models [23], TFF1 remains a possible target on the basis of the following evidence. TFF1 expression is known to be upregulated by E2 [33] and has been shown to be an inhibitor of breast cancer metastasis in an animal model [23]. More importantly, the majority of the published clinical observations have shown that TFF1-positive primary breast tumors have a better outcome profile [23], consistent with our finding that the wild-type alleles of the SNPs were associated with a decreased risk of poor survival. Chromatin conformation studies (for example, chromosome conformation capture (3C) [34]) will help determine whether this 21q22.3 ERE interacts with its target genes and regulates expression by acting as a long-range regulator. Interestingly, with our preliminary results detected by $3 \mathrm{C}$, we have identified a specific region within TFF1 which can form a secondary structure with the 21q22.3 ERE-associated sequence. This interaction is more obvious in ER + breast cancer cells than in ER - cells and can be enhanced by the addition of E2 (Additional file 2: Figure S2). No such interaction was detected between the 21q22.3 ERE-associated sequence and other genes (for example, TMPRSS3) within this region (unpublished observation).

In the same way that the effect of individual SNPs on cancer incidence is small, the polymorphic alleles of ERE-associated SNPs at 21q22.3 predispose carriers to only a moderately increased risk of poor survival. Thus, the significance of such SNPs depends not only on the effect of each SNP alone but also on the interaction between functionally related alleles of individual SNPs. Our finding showing that the association between survival and the 21q22.3 SNPs was significantly modified by the SNP tagging ESR1 and the SNP tagging the Rad21-containing block (Figures 4 and 6G) is in line with this suggestion. The observed interaction between ERE-associated SNPs and either the ESR1 SNP or the ER status of the tumor also provides evidence for the breast tumorigenic relevance of these ERE-associated SNPs.

In the present study, we identified genetic variations at 21q22.3 as important factors in susceptibility to breast cancer progression. We attempted to address the possibility of false-positives and the effects of multiple testing by demonstrating a significant $P$-value in the permutation test. Furthermore, the two independent cohorts of patients yielded consistent results. Together with the functional experiments in the present study, these associations suggest that $\operatorname{Rad} 21$ promotes the effect of ER $\alpha$ in activating expression of E2-responsive genes, such as TFF1, which affects patients' risk of poor survival. Expression quantitative trait locus-based analysis to examine if there is a link between 21q22.3 SNPs and expression of target genes in human populations is certainly warranted. More importantly, in our present study, we started with comprehensive genome-wide screening for ERE-associated loci that were significantly associated with survival status of the patient and demonstrated that some ERE-associated SNPs showed a significant association with survival of breast cancer patients (Figure 2). This suggests that these SNPs are not just important ones, but the most important ones, in determining susceptibility to breast cancer progression. As a result, in our ongoing study, on the basis of the individual contributions of these significant EREassociated SNPs to breast cancer progression, we are attempting to generate a genetic risk score that can predict the DFS of our patients. Our preliminary results show that the inclusion of data for these significant ERE-associated SNPs significantly increases the area under the receiver operating characteristic curve. This finding might be critical in the development of new therapeutic and diagnostic approaches for breast cancer.

\section{Conclusions}

The promise of personalized medicine, in which the associated risk and the course of diseases, as well as the efficacy of treatment protocols, may be predicted on the basis of a person's genotype, must been tempered with caution. Nevertheless, validated molecular tests to assess the patient's germline DNA already drive therapeutic decisionmaking [7]. On the basis of the well-documented role of ER in breast cancer progression, we explored whether genetic variations in EREs, the sequences bound by ER to activate the transcriptional regulation of target genes, are associated with breast cancer progression. Notably, the ERE sites genotyped have been shown to bind ER $\alpha$ in vivo using ChIP-based methods on a genome-wide scale, providing a unique opportunity to comprehensively examine putative ERE sites without depending on an a priori hypothesis. The SNPs at the 21q22.3 ERE were found to affect the binding of ER to ERE, leading to a difference in ER-regulated transcription, and to be significantly associated with OS and DFS. These findings support the idea that functional variants in the ER $\alpha$-regulating sequence at 21 q22.3 are important in determining breast cancer progression, as well as providing support for a role of ERE SNPs in breast cancer progression. 


\section{Additional files}

Additional file 1: Figure $\mathbf{S 1}$. Genes within the region $1,000 \mathrm{~kb} 5^{\prime}$ to $1,000 \mathrm{~kb} \mathrm{3'}$ of rs 2839494 in 21q22.3. (A) Exons (vertical red lines) and untranslated regions (blue) of all the genes and untranslated mRNAs (green) in this region, 14 of which have been reported to be involved in various tumorigenic mechanisms (B). (C) Estradiol (E2)/estrogen receptor (ER)-dependent expression of mRNAs for these genes detected by RT-qPCR in ER-positive (MCF-7) and ER-negative (MDA-MB-231) breast cancer cell lines. The results are normalized to those for $\beta$-actin mRNA. N.D., not done. ${ }^{*} P<0.05$ for differences between the conditions with E2 and without E2 in the same cell.

Additional file 2: Figure S2. Chromosome conformation capture (3C) suggests that the 21q22.3 ERE-associated sequence interacts with TFF sequence. (A) Schematic diagrams of the 21q22.3 region containing TFF1, TMPRSS3, 21q22.3 SNPs and restriction enzyme (EcoRI) sites and the primers of quantitative PCR (qPCR) used in 3C. (B) Hypothesized model showing that the 21q22.3 ERE-associated sequence, after binding by the E2-ERa-p21 complex, forms a secondary structure with a specific region within TFF1. After restriction enzyme digestion and sequence linking, QPCR was performed using the forward and reverse primers (that is, F-primer and R-primer shown in the figure). (C) Relative DNA amounts detected by qPCR and the interaction between 21q22.3 ERE-associated sequence and TFF1, measured by amplified qPCR product, are more significant in ER-positive breast cancer cell lines (that is, MCF-7 and T47D), than in ER-negative cells (that is, MDA-MB-231, HS578T and MDA-MB-453) and can be enhanced by the addition of E2 (detected in T47D).

\section{Abbreviations}

aOR: Adjusted odds ratio; ChIP: Chromatin immunoprecipitation; Cl: Confidence interval; DFS: Disease-free survival; ENCODE: Encyclopedia of DNA elements; ERE: Estrogen response element; ERa: Estrogen receptor a; GWAS: Genome-wide association studies; LD: Linkage disequilibrium; OS: Overall survival; SNP: Single-nucleotide polymorphism.

\section{Competing interests}

The authors declare that they have no competing interests.

\section{Authors' contributions}

CYS participated in the generation of the study concept and in the study design and coordination, and drafted the manuscript. The following authors made substantial contributions to the analysis, experiments and interpretation of data for the work. CNH and PEW carried out ERE site searches, genotyping and data analysis. HWC, YLH, WCC and LYH performed functional assays. The following authors made substantial contributions to the acquisition of the data: HMH, MFH and JCY carried out the participant recruitment and interpretation of clinical information. All authors participated in drafting the manuscript and revising it critically for important intellectual content. All authors agree to be accountable for all aspects of the study to ensure that questions related to the accuracy or integrity of any part of the work are appropriately investigated and resolved. All authors read and approved the final manuscript.

\section{Acknowledgements}

This work was supported by grants from the Institute of Biomedical Sciences, Academia Sinica, Taiwan.

\section{Author details}

'Institute of Biomedical Sciences, Academia Sinica, Academy Street, Taipei 115, Taiwan. ${ }^{2}$ Taiwan Biobank, Academia Sinica, Academy Street, Taipei 115, Taiwan. ${ }^{3}$ National Defense Medical College, Department of Surgery, Tri-Service General Hospital, Chenggong Road, Taipei 114, Taiwan. ${ }^{4}$ Cancer Center and Department of Surgery, Kaohsiung Medical University Chung-Ho Memorial Hospital, Tzyou 1st Road, Kaohsiung 804, Taiwan. ${ }^{5}$ College of Public Health, China Medical University, Hsueh-Shih Road, Taichung 404, Taiwan.

Received: 27 May 2014 Accepted: 2 October 2014

Published online: 09 October 2014

\section{References}

1. Yager JD, Davidson NE: Estrogen carcinogenesis in breast cancer. N Engl J Med 2006, 354:270-282.

2. Osborne CK, Schiff R: Estrogen-receptor biology: continuing progress and therapeutic implications. J Clin Oncol 2005, 23:1616-1622.

3. Green KA, Carroll JS: Oestrogen-receptor-mediated transcription and the influence of co-factors and chromatin state. Nat Rev Cancer 2007, 7:713-722.

4. Shang Y: Molecular mechanisms of oestrogen and SERMs in endometrial carcinogenesis. Nat Rev Cancer 2006, 6:360-368.

5. Bond GL, Hu W, Bond EE, Robins H, Lutzker SG, Arva NC, Bargonetti J, Bartek F, Taubert H, Wuerl P, Onel K, Yip L, Hwang SJ, Strong LC, Lozano G, Levine AJ: A single nucleotide polymorphism in the MDM2 promoter attenuates the p53 tumor suppressor pathway and accelerates tumor formation in humans. Cell 2004, 119:591-602.

6. Li LC, Chui RM, Sasaki M, Nakajima K, Perinchery G, Au HC, Nojima D, Carroll P, Dahiya R: A single nucleotide polymorphism in the E-cadherin gene promoter alters transcriptional activities. Cancer Res 2000, 60:873-876.

7. Yu JC, Hsiung CN, Hsu HM, Bao BY, Chen ST, Hsu GC, Chou WC, Hu LY, Ding SL, Cheng CW, Wu PE, Shen CY: Genetic variation in the genome-wide predicted estrogen response element-related sequences is associated with breast cancer development. Breast Cancer Res 2011, 13:R13.

8. Ross-Innes CS, Stark R, Teschendorff AE, Holmes KA, Ali HR, Dunning MJ, Brown GD, Gojis O, Ellis IO, Green AR, Ali S, Chin SF, Palmieri C, Caldas C, Carroll JS: Differential oestrogen receptor binding is associated with clinical outcome in breast cancer. Nature 2012, 481:389-393.

9. Lin CY, Vega VB, Thomsen JS, Zhang T, Kong SL, Xie M, Chiu KP, Lipovich L, Barnett DH, Stossi F, Yeo A, George J, Kuznetsov VA, Lee YK, Charn TH, Palanisamy N, Miller LD, Cheung E, Katzenellenbogen BS, Ruan Y, Bourque G, Wei CL, Liu ET: Whole-genome cartography of estrogen receptor a binding sites. PLoS Genet 2007, 3:e87.

10. Welboren WJ, van Driel MA, Janssen-Megens EM, van Heeringen SJ, Sweep FCGJ, Span PN, Stunnenberg HG: ChIP-Seq of ERa and RNA polymerase II defines genes differentially responding to ligands. EMBO J 2009, 28:1418-1428.

11. Ding SL, Yu JC, Chen ST, Hsu GC, Hsu HM, Ho JY, Lin YH, Chang CC, Fann SJ, Cheng CW, Wu PE, Shen CY: Diverse associations between ESR1 polymorphism and breast cancer development and progression. Clin Cancer Res 2010, 16:3473-3484.

12. Ding SL, Yu JC, Chen ST, Hsu GC, Kuo SJ, Lin YH, Wu PE, Shen CY: Genetic variants of $B L M$ interact with RAD51 to increase breast cancer susceptibility. Carcinogenesis 2009, 30:43-49.

13. Ding SL, Yu JC, Chen ST, Hsu GC, Shen CY: Genetic variation in the premature aging gene WRN: a case-control study on breast cancer susceptibility. Cancer Epidemiol Biomarkers Prev 2007, 16:263-269.

14. Taiwan Biobank. [https://taiwanview.twbiobank.org.tw/taiwanview/twbinfo.do] (accessed 14 October 2014).

15. Michailidou K, Hall P, Gonzalez-Neira A, Ghoussaini M, Dennis J, Milne RL, Schmidt MK, Chang-Claude J, Bojesen SE, Bolla MK, Wang Q, Dicks E, Lee A, Turnbull C, Rahman N, The Breast and Ovarian Cancer Susceptibility Collaboration, Fletcher O, Peto J, Gibson L, dos Santos Silva I, Nevanlinna H, Muranen TA, Aittomäki K, Blomqvist C, Czene K, Irwanto A, Liu J, Waisfisz Q, Meijers-Heijboer H, Adank $M$, et al: Large-scale genotyping identifies 41 new loci associated with breast cancer risk. Nat Genet 2013, 45:353-361

16. Zheng W, Zhang B, Cai Q, Sung H, Michailidou K, Shi J, Choi JY, Long J, Dennis J, Humphreys MK, Wang Q, Lu W, Gao YT, Li C, Cai H, Park SK, Yoo KY, Noh DY, Han W, Dunning AM, Benitez J, Vincent D, Bacot F, Tessier D, Kim SW, Lee MH, Lee JW, Lee JY, Xiang YB, Zheng Y: Common genetic determinants of breast-cancer risk in East Asian women: a collaborative study of 23637 breast cancer cases and 25579 controls. Hum Mol Genet 2013, 22:2539-2550.

17. Browning BL: PRESTO: rapid calculation of order statistic distributions and multiple-testing adjusted P-values via permutation for one and two-stage genetic association studies. BMC Bioinformatics 2008, 9:309.

18. Barrett JC, Fry B, Maller J: Haploview: analysis and visualization of LD and haplotype maps. Bioinformatics 2005, 21:263-265.

19. Stephens M, Smith NJ, Donnelly P: A new statistical method for haplotype reconstruction from population data. Am J Hum Genet 2001, 68:978-989.

20. Chu HW, Cheng CW, Chou WC, Hu LY, Wang HW, Hsiung CN, Hsu HM, Wu PE, Hou MF, Shen CY, Yu JC: A novel estrogen receptor-microRNA 190a-PAR-1pathway regulates breast cancer progression, a finding initially suggested 
by genome-wide analysis of loci associated with lymph-node metastasis. Hum Mol Genet 2014, 23:355-367.

21. Kelsey JL, Gammon MD, John EM: Reproductive factors and breast cancer. Epidemiol Rev 1993, 15:36-47.

22. Cheng YC, Hsiao FC, Yeh EC, Lin WJ, Tang CY, Tseng HC, Wu HT, Liu CK, Chen CC, Chen YT, Yao A: VarioWatch: providing large-scale and comprehensive annotations on human genomic variants in the next generation sequencing era. Nucleic Acids Res 2012,

40(Web Server issue):W76-W81.

23. Buache E, Etique N, Alpy F, Stoll I, Muckensturm M, Reina-San-Martin B, Chenard MP, Tomasetto C, Rio MC: Deficiency in trefoil factor 1 (TFF1) increases tumorigenicity of human breast cancer cells and mammary tumor development in TFF1-knockout mice. Oncogene 2011, 30:3261-3273.

24. Scott HS, Kudoh J, Wattenhofer M, Shibuya K, Berry A, Chrast R, Guipponi M, Wang J, Kawasaki K, Asakawa S, Minoshima S, Younus F, Mehdi SQ,

Radhakrishna U, Papasawas MP, Gehrig C, Rossier C, Korostishevsky M, Gal A, Shimizu N, Bonne-Tamir B, Antonarakis SE: Insertion of $\beta$-satellite repeats identifies a transmembrane protease causing both congenital and childhood onset autosomal recessive deafness. Nat Genet 2001, 27:59-63.

25. Lee YJ, Park D, Kim SY, Park WJ: Pathogenic mutations but not polymorphisms in congenital and childhood onset autosomal recessive deafness disrupt the proteolytic activity of TMPRSS3. J Med Genet 2003, 40:629-631.

26. The ENCODE Project Consortium: A user's guide to the Encyclopedia of DNA Elements (ENCODE). PLoS Biol 2011, 9:e1001046.

27. McEwan MV, Eccles MR, Horsfield JA: Cohesin is required for activation of MYC by estradiol. PLoS One 2012, 7:e49160.

28. Fugger $L, M c V e a n G$, Bell Jl: Genomewide association studies and common disease-realizing clinical utility. N Engl J Med 2012, 367:2370-2371.

29. Dunbier AK, Anderson H, Ghazoui Z, Folkerd EJ, A'Hern R, Crowder RJ, Hoog J. Smith IE, Osin P, Nerurkar A, Parker JS, Perou CM, Ellis MJ, Dowsett M: Relationship between plasma estradiol levels and estrogen-responsive gene expression in estrogen receptor-positive breast cancer in postmenopausal women. J Clin Oncol 2010, 28:1161-1167.

30. Lin Z, Reierstad S, Huang CC, Bulun SE: Novel estrogen receptor-alpha binding sites and estradiol target genes identified by chromatin immunoprecipitation cloning in breast cancer. Cancer Res 2007, 67:5017-5024.

31. Guerrero K, Wang Z, Bachvarova M, Gregoire J, Renaud MC, Plante M, Bachvarov D: A novel genome-based approach correlates TMPRSS3 overexpression in ovarian cancer with DNA hypomethylation. Gynecol Oncol 2012, 125:720-726.

32. Perry JK, Kannan N, Grandison PM, Mitchell MD, Lobie PE: Are trefoil factors oncogenic? Trends Endocrinol Metab 2008, 19:74-81.

33. Li Y, Sun L, Zhang Y, Wang D, Wang F, Liang J, Gui B, Shang Y: The histone modifications governing TFF1 transcription mediated by estrogen receptor. J Biol Chem 2011, 286:13925-13936.

34. Sajan SA, Hawkins RD: Methods for identifying higher-order chromatin structure. Annu Rev Genomics Hum Genet 2012, 13:59-82.

doi:10.1186/s13058-014-0455-

Cite this article as: Hsiung et al:: Functional variants at the 21q22.3 locus involved in breast cancer progression identified by screening of genome-wide estrogen response elements. Breast Cancer Research 2014 16:455.

\section{Submit your next manuscript to BioMed Central and take full advantage of:}

- Convenient online submission

- Thorough peer review

- No space constraints or color figure charges

- Immediate publication on acceptance

- Inclusion in PubMed, CAS, Scopus and Google Scholar

- Research which is freely available for redistribution 